\title{
Elsner, Daniela, Tim Engartner, Subin Nijhawan, und Nina Rodmann (2019): Politik \& Wirtschaft bilingual unterrichten
}

\author{
Frankfurt am Main: Wochenschau Verlag. 70 Seiten. 12,90€
}

\section{Alexander Wohnig}

Online publiziert: 14. April 2020

(C) Der/die Autor(en) 2020

Im Rahmen des Projekts PolECulE (Politics, Economics \& Culture in English), das an den Fachdidaktikprofessuren des Instituts für England- und Amerikastudien sowie des Instituts für Politikwissenschaft der Goethe-Universität Frankfurt am Main durchgeführt wird, ist eine kleine Publikation entstanden, in der auf der Basis des hessischen Kerncurriculums für das Schulfach Politik und Wirtschaft (PoWi) für die Sekundarstufe I ein Orientierungsrahmen für den bilingualen Politik- und Wirtschaftsunterricht vorgelegt wird. Bilinguales Lernen wird dabei verstanden als eine Verknüpfung von sachfachlichem Lernen - in diesem Fall Inhalte des Kerncurriculums Politik und Wirtschaft - sowie (fremd-)sprachlichem Lernen. Überzeugend an dem vorliegenden Konzept ist vor allem die Erweiterung des Fachs und seiner Inhalte um den Aspekt der Kultur, der durch den Einbezug des Fremdsprachenlernens hinzutritt, sowie die Definition des Fachs als eines mit integrativem Charakter. Dabei wird davon ausgegangen, dass politisch-ökonomische Fragestellungen immer eine integrative Aufarbeitung verlangen, was der aktuell in vielen Bundesländern beobachtbaren Tendenz entgegenwirkt, Separationsfächer zu etablieren. (So wurde und wird in einigen Bundesländern das Schulfach Wirtschaft eingeführt.)

Das Ziel des Projekts, dem die Publikation entstammt, ist es, einem bilingualen Unterricht im Sinne von Politics, Economics \& Culture den Weg zu bahnen. Dieses Ziel wird dadurch unterstützt, dass LehrerInnen eine praktische „Anleitung“ in Form eines curricularen Orientierungsrahmens erhalten, der direkt an die hessischen Kerncurricula für die Sekundarstufe I anschließt und damit auch unmittelbar umsetzbar ist. Die Kerncurricula wurden demensprechend erweitert, sodass die Inhaltsfelder und Kompetenzerwartungen des Fachs PoWi mit Aspekten des fremdsprachlichen Lernens verbunden wurden. Was dies bedeutet, lässt sich exemplarisch in der Dar-

\footnotetext{
A. Wohnig $(\bowtie)$

Philosophische Fakultät, Universität Siegen, Siegen, Deutschland

E-Mail: alexander.wohnig@uni-siegen.de
} 
stellung der Kompetenzbereiche des bilingualen PoWi-Unterrichts zeigen: Neben die Analyse-, die Urteils-, die Handlungs- und die Methodenkompetenz - allesamt „klassische“ Ziele politischer Bildung - treten die inter- bzw. transkulturelle und die (fremd-)sprachlich-kommunikative Kompetenz. Diese sechs Kompetenzbereiche kulminieren in der globalen Diskurskompetenz, das übergreifende, interdisziplinäre Kompetenzziel des bilingualen PoWi-Unterrichts. Kern und Ziel dieses Kompetenzmodells ist die Eröffnung von Perspektiven, ,die kosmopolitisches Denken im Hinblick auf eine nachhaltige Entwicklung fördern“ (S. 8), womit eine „Basis der Völkerverständigung und Friedenserziehung“ (S. 10) gelegt werde. Mündige BürgerInnen werden als eigenständig denkende und zur Teilnahme am öffentlichen Diskurs befähigte Menschen definiert, die eine ,kosmopolitische Perspektive [...] in der (trans-)nationalen Zivilgesellschaft“" (S. 11) einnehmen können. So wird kosmopolitisches Denken als Ziel und als Form einer Perspektivenerweiterung weg von einer rein lokalen Perspektive des bilingualen PoWi-Unterrichts definiert: „Im Bereich des kulturellen Lernens soll den Lernenden eine kosmopolitische Perspektive eröffnet werden, aus der heraus sie die multi- und transkulturelle Zivilgesellschaft in ihrer perspektivischen Vielfalt erfassen und verstehen lernen.“ (S. 26) Im Zentrum der inter- bzw. transkulturellen Kompetenz steht sodann die „Unterstreichung von Gemeinsamkeiten [von Kulturen, AW], was für die Herausbildung einer kosmopolitischen Perspektive unerlässlich ist“" (S. 28).

Die methodischen Überlegungen zum bilingualen PoWi-Unterricht beziehen sich zum einen auf die didaktische Organisation des Gebrauchs der beiden Sprachen im Unterricht (hier Deutsch und Englisch) als auch auf einen konkreten Vorschlag zur Einbettung in den Unterricht mithilfe eines Planspiels. Das Planspiel wird mit zweisprachigen Materialien in der Publikation aufbereitet (mitgeliefert werden u.a. Reflexionsfragen, Rollenkarten, Materialien zur Vorbereitung auf das Rollenspiel, Beschreibung des Vorgehens usw.) und kann somit von LehrerInnen im Schulunterricht, aber auch von PädagogInnen in der außerschulischen politischen Bildung durchgeführt werden (S. 54-68).

Auf der Grundlage aktueller didaktischer Forschung zum bilingualen Unterricht schlagen die AutorInnen vor, Sprachwechsel in den Unterricht einzubauen, sodass die Lehrperson den Unterricht dergestalt organisiert, dass zwischen deutschsprachigen und englischsprachigen Phasen ein Wechsel stattfindet. Auch dafür werden unterschiedliche praxistaugliche Modelle skizziert (S. 48-53).

Mit dem vorliegenden Orientierungsrahmen werden sehr hohe Ziele und Erwartungen an den bilingualen PoWi-Unterricht geknüpft. Es stellt sich die Frage, ob die Definition mündiger BürgerInnen sinnvoll mit kosmopolitischen Einstellungen verbunden werden sollte, oder ob SchülerInnen nicht auch - so eine beliebte Gegenüberstellung in den aktuellen politikwissenschaftlichen Debatten zum Populismus - kommunitaristische Urteile entwickeln können, wenn sie politische, ökonomische und kulturelle Probleme mithilfe einer kosmopolitischen Perspektive betrachtet haben. Für die kosmopolitische Perspektive spricht die Zeitdiagnose, die die AutorInnen zum Anlass ihres Projekts nehmen: die zunehmende Globalisierung von Wirtschaft, Gesellschaft und Politik sowie die globalen Auswirkungen von politischen, gesellschaftlichen und wirtschaftlichen Entscheidungen und darauf basierendem Handeln. Unter den Bedingungen einer solchen globalen Vernetzung erscheint 
es sinnvoll Englisch als Lingua franca in Bezug auf politisch-ökonomische Bildungsbemühungen zu fördern, da Sprache ein ,grundlegendes Werkzeug zur sinnstiftenden Teilhabe an Diskursen jedweder Art“ ist und „damit als Grundlage für die Aneignung der [...] Analyse-, Urteils-, Handlungs- und Methodenkompetenz“ (S. 28) verstanden werden kann. Eine Reflexion des Umgangs mit sozioökonomischer sowie Bildungsungleichheit und ihren Wirkungen auf einen solchen Unterricht wäre für den weiteren Verlauf des Projekts wünschenswert.

Funding Open Access funding provided by Projekt DEAL.

Open Access Dieser Artikel wird unter der Creative Commons Namensnennung 4.0 International Lizenz veröffentlicht, welche die Nutzung, Vervielfältigung, Bearbeitung, Verbreitung und Wiedergabe in jeglichem Medium und Format erlaubt, sofern Sie den/die ursprünglichen Autor(en) und die Quelle ordnungsgemäß nennen, einen Link zur Creative Commons Lizenz beifügen und angeben, ob Änderungen vorgenommen wurden.

Die in diesem Artikel enthaltenen Bilder und sonstiges Drittmaterial unterliegen ebenfalls der genannten Creative Commons Lizenz, sofern sich aus der Abbildungslegende nichts anderes ergibt. Sofern das betreffende Material nicht unter der genannten Creative Commons Lizenz steht und die betreffende Handlung nicht nach gesetzlichen Vorschriften erlaubt ist, ist für die oben aufgeführten Weiterverwendungen des Materials die Einwilligung des jeweiligen Rechteinhabers einzuholen.

Weitere Details zur Lizenz entnehmen Sie bitte der Lizenzinformation auf http://creativecommons.org/ licenses/by/4.0/deed.de. 